\title{
An all-oral and interferon-free future for HCV therapy?
}

The current standard of care (SOC) for hepatitis $\mathrm{C}$ virus (HCV) infection involves treatment with ribavirin and pegylated interferon (PEG-IFN). This stimulates the immune system rather than targeting the virus directly, and is associated with severe side effects. Earlier this year, the first two direct-acting antiviral agents (DAAs), the protease inhibitors telaprevir (Incivek; Vertex/Johnson \& Johnson) and boceprevir (Victrelis; Merck), were approved by the US Food and Drug Administration; however, these still have to be administered in combination with ribavirin and PEG-IFN.

Now, data presented at the American Association for the Study of Liver Diseases (AASLD) 2011 Annual Meeting show that the goal of achieving an all-oral and IFN-free treatment regimen is achievable and might be close. In particular, clinical trial results with HCV polymerase inhibitors have generated excitement. Michael Manns, Medical School of Hannover, Germany, explains: "The advantage of nucleoside polymerase inhibitors is that they are pan-genotypic, which means that they show activity against various - maybe all - HCV subtypes, which is in contrast to most $\mathrm{HCV}$ protease inhibitors. Moreover, they have a high genetic barrier to resistance, strong antiviral potency and a favourable safety profile." He is particularly excited about the uracil nucleotide analog polymerase inhibitor PSI-7977 (Pharmasset). “A two-drug combination, PSI-7977 plus ribavirin, was shown to be successful for HCV genotype 2 and 3 patients and another study with PSI-7977 in combination with PEG-IFN and ribavirin showed promising results for HCV genotype 1, which is currently more difficult to treat."

Pharmasset announced the initiation of a Phase III programme that will evaluate PSI-7977 in a 12-week, all-oral, IFN-free regimen in combination with ribavirin.

Another nucleoside polymerase inhibitor, INX-189 (Inhibitex), was shown to achieve robust and dose-dependent decreases in HCV RNA levels after 7 days of monotherapy or in combination with ribavirin in treatment-naive patients with chronic HCV genotype 1 . The company announced that it will now expand its clinical development programme to include IFN-free combinations of INX-189 with other DAAs in patients with HCV genotype 1, 2 and 3.

Pharmasset will test PSI-7977 in combination with the small-molecule NS5A replication complex inhibitor BMS-790052 (Bristol-Myers Squibb). BMS-790052 showed promising results in combination with the protease inhibitor BMS-650032 in an early-stage, all-oral, IFN-free combination study, achieving a $100 \%$ cure rate in all nine Japanese patients with HCV genotype $1 \mathrm{~b}$ who completed treatment. Manns points out that this provides proof of concept for all-oral IFN and ribavirin-free anti-HCV therapies.

Finally, Roche and Anadys Pharmaceuticals have announced a merger agreement, in which Roche acquires Anadys for US\$230 million in an all-cash deal. This follows promising data for Anadys's setrobuvir (ANA598), a non-nucleoside oral smallmolecule HCV polymerase inhibitor that is currently in a Phase II study in combination with PEG-IFN and ribavirin. Again, the aim for this drug is to ultimately form part of an IFN-free oral DAA treatment regimen.

"IFN has numerous side effects and many patients cannot be included in SOC treatments because of exclusion criteria," notes Ralf Bartenschlager, University of Heidelberg, Germany. "These problems are circumvented with combinations of DAAs and, therefore, more patients can be treated and side effects are lower." He sees the future of IFN-free anti-HCV treatments in DAA combinations such as an NS5A inhibitor (as these have shown high potency), a nucleoside polymerase inhibitor and eventually a second-generation protease inhibitor, which has higher potency, fewer side effects and less genotype dependency compared with the first-generation protease inhibitors.

"An IFN-free (and also ribavirin-free) therapy is the main goal, and given the very promising data from clinical trials, this seems to be realistic. The prospects for curing a patient with $\mathrm{HCV}$ by an IFN-free treatment regimen will become very high," he concludes. 